\section{Doença de Hirschsprung: experiência com uma série de 55 casos}

\section{Hirschsprung's disease: experiment with a serie of 55 cases}

Maria Auxiliadora Monteiro Villar 1

Monica de Paula Jung 2

Leila Cabral de Almeida Cardoso 3

Maria Helena Cabral de Almeida Cardoso 4

Juan Clinton Llerena Junior 5

,3,4,5 Departamento de Genética. Instituto Fernandes Figueira Fundação Oswaldo Cruz. Av Rui Barbosa, 716, Flamengo. Rio de Janeiro, RJ, Brasil. CEP: 22.250-020.

E-mail: cardosomhca@iff.fiocruz.br

2 Instituto Estadual de Diabetes e Endocrinologia Luiz Capiglione - IEDE. Rio de Janeiro, RJ.

\section{Abstract}

Objectives: to analyze clinical features of patients with Hirschprung's Disease (HD).

Methods: a retrospective institutional case study was carried out using the medical records of patients with HD attending the Fernandes Figueira Institute between 1993 and 2003.

Results: out of a total of 55 patients, $98 \%$ presented symptoms on birth, $47.2 \%$ of whom were diagnosed during the neonatal period; in $88.9 \%$ of cases the barium enema was conclusive; $69 \%$ had short segment HD; $16.3 \%$ Down's Syndrome; $15.2 \%$ other congenital anomalies; $40 \%$ were screened for RET mutations associated with multiple endocrine neoplasias (MEN2A), although none were detected; $63.6 \%$ had staged pull-through surgery on the colon or ileum; $12.72 \%$ primary transanal endorectal pullthrough surgery; the main complications arising from surgery were sepsis, enterocolitis and obstruction of the intestines; abnormal bowel movements were detected years after the surgery; the mortality rate was $9.25 \%$, the causes of death being post-operal enterocolitis and sepsis.

Conclusion: although patients presented symptoms on birth, most were diagnosed at a later stage. Enterocolitis was the main cause of death. Abnormal bowel movements frequently occurred, requiring prolonged follow-up. Although rare, the association with MEN2A needs to be investigated owing to the highly aggressive nature of the disease. The clinical and genetic heterogeneity of HD necessitates the involvement of a multidisciplinary team.

Key words Hirschsprung Disease, Clinical Aspects, Child Health

\section{Resumo}

Objetivos: analisar aspectos clínicos de pacientes com Doença de Hirschprung (DH).

Métodos: realizou-se estudo de caso institucional, retrospectivo, via revisão de prontuários de pacientes com DH atendidos no Instituto Fernandes Figueira entre 1993 e 2003.

Resultados: Em um total de 55 pacientes, 98\% apresentaram sintomas neonatais, sendo $47,2 \%$ diagnosticados nesse periodo; em $88,9 \%$ o enema baritado foi conclusivo; $69 \%$ tinham DH de segmento curto; $16,3 \%$ sindrome de Down; 15,2\% outras anomalias congênitas; $40 \%$ foram rastreados para mutações RET associadas a neoplasias endócrinas múltiplas (MEN2A), não sendo detectada nenhuma; 63,6\% fizeram abaixamento estagiado do colon/íleo; $12,72 \%$ abaixamento endoretal transanal primário; as principais complicações cirúrgicas foram sepse, enterocolite e obstrução intestinal; distúrbios da defecação foram detectados anos pós-cirurgia; a taxa de letalidade foi 9,25\%; os óbitos relacionaram-se a enterocolite e sepse pós-operatórias.

Conclusão: embora apresentasse sintomas neonatais, a maioria dos pacientes foi diagnosticada tardiamente. Enterocolite foi a principal causa de morbimortalidade. Distúrbios da defecação ocorrem com frequência, demandando follow-up prolongado. Embora rara, a associação com MEN2A precisa ser investigada devido à agressividade da doença. A heterogeneidade clínica e genética da DH exige atuação de equipe multidisciplinar.

Palavras-chave Doença de Hirschsprung, Aspectos Clínicos, Saúde da Criança 
Introdução

A Doença de Hirschprung (DH) ou megacólon congênito caracteriza-se pela ausência de células gangliônicas no plexo submucoso e mioentérico, em variadas porções do intestino, sendo causa relativamente comum de obstrução intestinal em neonatos e megacólon em crianças e adultos. ${ }^{1}$ Sua incidência é de cerca de 1:5000 nascidos vivos e acomete predominantemente o sexo masculino na razão de $4: 1 .^{2}$

Considera-se como DH de segmento curto (80\% dos casos), quando o segmento agangliônico não ultrapassa o cólon sigmóide, ou de segmento longo (20\% dos casos) quando ultrapassa o colón sigmóide podendo afetar todo o cólon - anglangliode colônica total (ACT), ou mesmo todo o intestino - aganglionose intestinal total (ATI).3-4 Ocorre, em geral, como defeito isolado, mas pode se associar a outras síndromes genéticas, sendo a associação mais frequente com a síndrome de Down e a mais grave com neoplasias endócrinas múltiplas tipo 2A (MEN2A).5-6 Também pode se associar com anomalias congênitas isoladas adicionais.2-7 É um defeito congênito, com padrão de herança complexo e multigênico influenciada pelo sexo. Nove genes de suscetibilidade à doença já foram mapeados e, entre eles, o gene RET é considerado o principal.2-7

A DH vem despertando interesse no meio científico por seu caráter múltiplo no que diz respeito às suas manifestações clínicas, etiologia e associação com outras anomalias genéticas. A proposta apresentada neste trabalho pretende contribuir para ampliar o conhecimento sobre tal complexidade, na medida em que se volta para analisar dez anos de experiência multidisciplinar no atendimento a pacientes com a doença. Trata-se, particularmente, de reunir numa série de casos a prática de cirurgiões, pediatras, geneticistas clínicos, radiologistas e patalogistas para dar conta dos variados aspectos envolvidos no diagnóstico e tratamento da $\mathrm{DH}$, dentro de uma mesma instituição brasileira de referência e, sobretudo, reforçar a necessidade de acompanhamento das complicações associadas a curto, médio e longo prazo.

O objetivo deste artigo é apresentar a experiência com a Doença de Hirschsprung (DH) em uma unidade terciária, o Instituto Fernandes Figueira (IFF) da Fundação Oswaldo Cruz (FIOCRUZ), descrevendo o diagnóstico e o tratamento oferecido aos pacientes que foram submetidos à intervenção cirúrgica, em função da $\mathrm{DH}$, inclusive investigando a presença de mutações no exon 10 do proto-oncogene RET que indicassem a associação com
MEN2A.

\section{Métodos}

Durante o ano de 2003 foram coletados dados para a realização de um estudo descritivo, do tipo série de casos, através do levantamento retrospectivo dos prontuários de pacientes atendidos nos Departamentos de Cirurgia Pediátrica e Pediatria do Instituto Fernandes Figueira (IFF/FIOCRUZ), no período de janeiro de 1993 a agosto de 2003. Os critérios de inclusão foram ter o diagnóstico histopatológico da Doença de Hirschsprung realizado no IFF. As lacunas encontradas foram preenchidas via reavaliação de pacientes que responderam favoravelmente à solicitação e compareceram ao hospital. Foram analisados 55 casos representando a totalidade dos pacientes atendidos no período recortado.

Todos os pacientes foram avaliados pela equipe médica do Departamento de Cirurgia Pediátrica e já haviam se submetido à biópsia retal ou laparotomia, tendo a doença confirmada pela avaliação histológica do material obtido. Durante o ato cirúrgico foi realizada biópsia de congelação para se determinar o nível da aganglionose. A confirmação diagnóstica foi através do exame histopatológico da peça cirúrgica, utilizando-se rotineiramente a técnica da coloração pela hematoxilina-eosina. Não houve mudanças significativas na equipe e mantiveram-se as técnicas de diagnóstico, de acordo com o padrãoouro estabelecido há décadas pela literatura especializada. 8 Os pacientes após alta cirúrgica foram acompanhados no Ambulatório de Pediatria e de Cirurgia Pediátrica.

Para organização e tabulação dos dados foi utilizado o programa de planilha eletrônica Excel. Foi realizada a análise de frequência, também se usando o Excel. Paralelamente, foram feitos arquivos individualizados, onde se registrou o estudo de cada caso.

A investigação de mutações de ponto associadas a MEN2A foi realizada no Instituto Nacional do Câncer. Entre os pacientes estudados, 22 consentiram em serem rastreados. As amostras de DNA de sete pacientes foram analisadas pelo sequenciamento manual, de acordo com o protocolo descrito no kit Thermo Sequence Radiolabeled Terminator Cycle Sequencing-USB ${ }^{\mathrm{TM}}$, e 15 pelo método de rastreamento de mutações por gel de eletroforese com gradiente de desnaturação (DGGE).

Esta pesquisa foi aprovada pelo Comitê de Ética em Pesquisa do IFF/FIOCRUZ sob o parecer $n^{\circ}$ $159 / 02$ 


\section{Resultados}

Entre janeiro de 1993 e agosto de 2003 foram atendidos, no IFF, um total de 55 pacientes que compuseram a série estudada. Em 2003, seis haviam falecido, dois haviam perdido o contato com o IFF e 41 estavam com o trânsito intestinal reconstruído.

Entre os pacientes estudados, 38 tinham DH de segmento curto $(69 \%)$ e 17 de segmento longo (31\%), sendo seis com ACT (11\%) e dois com ATI $(3,6 \%)$. A relação entre os sexos foi de $6,6: 1$ para a $\mathrm{DH}$ de segmento curto e de 1,4:1 para a $\mathrm{DH}$ de segmento longo, sempre a favor do sexo masculino. Entre todos os pacientes, nove (16,3\%) tinham síndrome de Down e sete $(15,2 \%)$ possuíam ano- malias isoladas adicionais, dos quais dois apresentavam mais de um tipo de malformação (Tabela 1).

Todos os pacientes, exceto um, apresentaram manifestações no período neonatal, sendo a tríade de manifestações mais frequentemente observada composta por retardo na eliminação de mecônio, distensão abdominal progressiva e vômitos. Retardo na eliminação de mecônio associado a outros sintomas apresentou-se em $60 \%$ dos casos, ainda que isoladamente só tenha aparecido em 7,2\%. Da mesma forma a distensão abdominal progressiva foi registrada em $74,54 \%$ dos casos, mas isoladamente apenas em 3,63\%. Vômitos só se revelaram conjugados a outros sintomas, conforme se observa na Tabela 2.

\section{Tabela 1}

\begin{tabular}{llll}
\hline $\begin{array}{l}\text { Pacientes com Doença de Hirschsprung associada à Síndrome de Down ou a anomalias isoladas. } \\
\text { de casos }\end{array}$ & $\begin{array}{l}\text { Extensão da } \\
\text { Aganglionose }\end{array}$ & Síndromes Associadas & $\begin{array}{l}\text { Anomalias Congênitas } \\
\text { Isoladas }\end{array}$ \\
\hline 1 & Segmento longo & - & Rim ferradura, pé torto \\
2 & Segmento curto & - & Sistema urinário \\
1 & Segmento curto & - & Estenose anal \\
1 & Segmento longo & - & Agenesia renal esquerda \\
1 & Segmento curto & - & Criptorquidia e ascite quilosa \\
1 & Segmento curto & Cisto temporal não hipertensivo \\
5 & Segmento curto & Síndrome de Down & - \\
4 & Segmento longo & Síndrome de Down & - \\
\hline
\end{tabular}

\section{Tabela 2}

Frequência dos sintomas de apresentação no período neonatal.

\begin{tabular}{lcc}
\hline Sintomas & Número de casos & $\%$ \\
\hline Sintomas isolados & & \\
$\quad$ Distensão abdominal & 2 & 3,63 \\
Retardo na eliminação de mecônio & 4 & 7,27 \\
Constipação & 4 & 7,27 \\
Enterocolite & 4 & 7,27 \\
Perfuração Intestinal & 1 & 1,81 \\
Total & 15 & 27,25 \\
Sintomas conjugados & & \\
Retardo na eliminação de mecônio, distensão abdominal e vômitos & 22 & 40,00 \\
Retardo na eliminação de mecônio e distensão abdominal & 7 & 12,72 \\
Constipação com distensão abdominal & 5 & 9,09 \\
Distensão abdominal e vômitos & 5 & 9,09 \\
Total & 39 & 70,90 \\
\hline
\end{tabular}

Uma entre as 55 crianças não apresentou sintomas no período neonatal. 
Em 45 crianças $(81,8 \%)$ o resultado do enema baritado sugeriu DH e em 10 pacientes $(18,2 \%)$ o exame foi contraindicado devido às suas condições clínicas. Considerando apenas a população de recém-natos (29), o enema baritado foi feito em 22 , mostrando-se conclusivo em 17 (77,2\%).

No período estudado aconteceram seis óbitos, sendo cinco diretamente relacionados à $\mathrm{DH}$ e o um outro por leucemia monocítica aguda em um paciente com síndrome de Down. Portanto, excluindo-se da série este último, a taxa de letalidade calculada da doença, no IFF, considerando a totalidade já referida de 55 pacientes, foi de $9,25 \%$. Entre os cinco casos de óbito diretamente relacionados à $\mathrm{DH}$, todos haviam sido transferidos de outros hospitais durante o período neonatal, com média de idade de 6,4 dias (variando entre um e 17 dias). Todos apresentavam DH de segmento longo e faleceram devido à enterocolite e sepse no período pós-operatório.

Quanto ao tratamento cirúrgico, dos 55 pacientes estudados, $22(40 \%)$ tiveram enterocolite antes da primeira cirurgia para $\mathrm{DH}$.

$\mathrm{Na}$ maioria dos pacientes foi realizado colostomia ou ileostomia antes da reconstrução definitiva do trânsito intestinal. Apenas sete pacientes $(12,7 \%)$ foram submetidos a abaixamento endoretal primário, pela via transanal, tendo o primeiro ocorrido em março de 2000. Desde então essa é a tendência do serviço, utilizando-se o procedimento no período neonatal, sempre que as condições clínicas permitem.

As complicações precoces, após a realização de uma ostomia descompressiva, apresentadas pelos pacientes atendidos no IFF no período recortado pela pesquisa, estão listadas na Tabela 3 e as que mais frequentemente seguiram o reparo definitivo foram sepse $(15,5 \%)$, enterocolite $(20,7 \%)$, infecção de ferida operatória $(13,3 \%)$, abscesso de parede abdominal $(11,1 \%)$ e obstrução intestinal precoce $(11,1 \%)$.

Tabela 3

\begin{tabular}{|c|c|c|c|}
\hline \multirow[t]{2}{*}{ Complicações } & \multicolumn{2}{|c|}{ Ostomias $(\mathrm{N}=48)$} & \multirow[t]{2}{*}{ Extensão da aganglionose/número de casos } \\
\hline & $\mathbf{n}$ & $\%$ & \\
\hline Enterocolite & 6 & 12,5 & Retosigmóide/01 caso; ACT/04 casos; ATI/01 caso \\
\hline Sepse & 4 & 8,3 & ACT/03 casos; ATI/01 caso \\
\hline Sangramento (borda colostomia) & 1 & 2,0 & Retosigmóide/01 caso \\
\hline Obstrução intestinal & 1 & $2,0 *$ & ACT/01 caso \\
\hline \multirow[t]{2}{*}{ Abscesso de parede abdominal } & 2 & 4,1 & Retosigmóide/01 caso; Sigmóide/01 caso \\
\hline & & & Colón Descendente/02 casos; ACT/01 caso; \\
\hline Prolapso da ostomia & 4 & 8,3 & Retosigmóide/01 caso \\
\hline Descência anastomótica & 1 & 2,0 & Retosigmóide/01 caso \\
\hline Hemorragia digestiva alta (úlcera) & 1 & 2,0 & Retosigmóide/01 caso \\
\hline
\end{tabular}

* Colostomia realizada em segmento agangliônico, posteriormente foi diagnostica ACT e realizada ileostomia.

Na série investigada todos os pacientes que apresentaram sepse após a cirurgia definitiva tinham ou ACT ou ATI.

No que tange ao prognóstico funcional, 23 dos 41 pacientes com o trânsito intestinal reconstruído não puderam ser avaliados pois tinham menos de três anos de idade e/ou sofrido rebaixamento há menos de um ano. Isto porque distúrbios da defecação ocorrem quer pela tenra idade de crianças que ainda não controlam completamente o esfíncter anal, quer pelo pouco tempo decorrido pós cirurgia, uma vez que depois da ressecção do reto não há mais o relaxamento do esfíncter interno quando a distensão intestinal acontece perto da anastomose; ela só reaparece passados muitos meses, em função da penetração transanastomótica de axônios que proliferam. ${ }^{9}$ Os 18 pacientes restantes evoluíram com distúrbios da defecação, tais como constipação grave necessitando de lavagem intestinal com frequência, cólicas, dor à evacuação, flatulência, soiling, encoprese, incontinência fecal e obstrução intestinal. Entre estes, cinco apresentaram as seguintes compli- 
cações mecânicas: permanência de segmento colônico agangliônico, permanência de coto retal longo e hipertonia de esfíncter anal. Após a correção cirúrgica das complicações houve resolução da sintomatologia em três pacientes e melhora significativa nos outros dois. Os outros 13 evoluíram com distúrbios da defecação sem que a investigação denunciasse qualquer complicação mecânica. A maioria melhorou ao longo do tempo com o manuseio dietético. Cinco deles, contudo, ainda apresentam soiling e raros episódios de encoprese, anos após a reconstrução do trânsito.

Nenhuma mutação no exon 10 do gene RET associada à MEN2A foi encontrada nos 22 pacientes $(40 \%)$ rastreados.

\section{Discussão}

A série do IFF apresentou uma frequência maior de DH de segmento longo do que a média da literatura sobre $\mathrm{DH}$, refletindo a característica terciária do Hospital que possui um Departamento de Cirurgia Pediátrica, inclusive com uma Unidade de Terapia Intensiva Neonatal Cirúrgica.

Concordando com dados da literatura especializada, 2 na amostra estudada houve um predomínio de casos de DH de segmento curto no sexo masculino, a forma mais comum de apresentação da doença, aproximando-se de 1:1 na DH de segmento longo. Isto reflete o fato da DH ser uma doença de etiologia multifatorial e predominante no sexo masculino. Dentro do padrão de doença genética de etiologia multifatorial e influenciada pelo sexo, é no indivíduo cujo sexo é menos acometido que a doença se manifesta de forma mais grave.10 Por essa razão, na série estudada, quando a DH foi de segmento longo a razão entre sexo masculino versus feminino reduziu-se para 1,4:1.

A associação da DH com outras anomalias isoladas é relativamente frequente, variando entre $5 \%$ e $30 \%, 2$ e deve ser investigada, demandando a necessidade de realização de exames, como a ultrassonografia renal, ecocardiograma e radiografia de esqueleto em todos os pacientes. Em pacientes com $\mathrm{DH}$, a ocorrência de displasia ou agenesia renal, cardiopatia e defeitos de fusão vertebral tem sido maior do que a esperada na população geral. ${ }^{2-4,7}$ Neste estudo, 15,2\% dos pacientes com DH, sem outras síndromes, apresentaram anomalias associadas, sendo que a maioria delas acometeu o sistema urinário. Esse resultado está de acordo com o estudo de algumas das séries de casos publicados na literatura,2,11 ressaltando-se, contudo, que como não houve a investigação sistemática de anomalias isoladas adicionais pelos exames referidos, a frequência detectada pode ter sido subestimada.

Corroborando os resultados de outros estudos, 4 os sintomas da DH se manifestaram no período neonatal na maioria dos casos da série estudada. Entretanto, em que pese presença de sintomas neonatais, o diagnóstico neonatal foi realizado em apenas $47,2 \%$ dos casos, um percentual abaixo do encontrado em outros estudos realizados a partir da década de 80 , mostrando que $90 \%$ dos pacientes com DH tiveram o diagnóstico firmado ainda no período neonatal.8,12 Essa defasagem entre os resultados do presente estudo e os dados da literatura quanto à confirmação diagnóstica de DH no período neonatal, se deve provavelmente ao encaminhamento tardio dos casos aos serviços de maior complexidade, possivelmente devido a duas situações: a deficiência da rede básica de serviços de saúde do Estado do Rio de Janeiro, que nem sempre possui infraestrutura para a investigação diagnóstica dos casos de DH no período neonatal e o desconhecimento da doença por parte de neonatologistas e pediatras. O retardo no diagnóstico e tratamento da $\mathrm{DH}$ são fatores que contribuem para piora do prognóstico, pois propiciam o surgimento da enterocolite.13,14 Sendo assim, embora não seja possível afirmar, sugere-se que tais fatores possam ter contribuído para os elevados percentuais de enterocolite no pré-operatório na série estudada (40\%).

$\mathrm{Na}$ série de casos estudada, o coeficiente de letalidade pela doença foi de $9 \%$ e entre os casos que evoluíram para o óbito, dois pacientes apresentavam $\mathrm{DH}$ com ACT e dois com ATI, as formas mais raras e geralmente fatais da doença.

Pacientes com ACT têm um significativo aumento da morbimortalidade, necessitando muitas vezes de nutrição parenteral prolongada e acesso venoso profundo. Na série estudada todos os pacientes com ACT receberam nutrição parenteral através de acesso venoso profundo. Nas situações nas quais a aganglionose se estende muito além do cólon, o prognóstico piora devido à deficiência de absorção de nutrientes no intestino delgado. A mortalidade é alta e geralmente se dá em consequência de sepse e/ou insuficiência hepática. ${ }^{14,15}$

A acurácia dos exames radiológicos no diagnóstico de DH na presente série de casos, de $81,8 \%$, é satisfatória quando comparada, por exemplo, à estabelecida $(67,9 \%)$ por estudos como o realizado por Singh et al.13 Entretanto, o retardo no encaminhamento dos doentes impossibilitou a realização do exame em alguns casos.

Quanto ao exame histopatológico, feito rotineiramente no IFF, utiliza-se a técnica da 
coloração pela hematoxilina-eosina, a qual impede a detecção de outras doenças que podem cursar com a $\mathrm{DH}$, tal como a displasia neuronal intestinal, interferindo negativamente no prognóstico e manuseio dos doentes após a cirurgia definitiva.

Com relação ao tratamento e as complicações da $\mathrm{DH}$, a maioria das séries estudadas ${ }^{3}$ revela uma parcela significativa de pacientes submetidos ao tratamento cirúrgico com prognóstico insatisfatório no que tange à função intestinal e tais resultados não parecem estar relacionados à técnica cirúrgica utilizada, acarretando prejuízos emocionais e sociais importantes.3,16-17 Enterocolite, constipação e incontinência fecal representam as principais complicações pós-operatórias. Distúrbios da motilidade intestinal, tais como urgência, dor e soiling também não são incomuns. Com o passar do tempo, a maioria dos indivíduos melhora a continência, mas alguns persistem incontinentes até o final da adolescência. 17-19

Em relação à função intestinal, na série estudada não há registro de follow-up prolongado para todos os portadores de DH que permita estimar a frequência de cada tipo de complicação tardia relacionada à doença, ou avaliar o prognóstico

\section{Referências}

1. Kusafutka T, Wang Y, Puri P. Hum Mol Genet. 1996; 5: 3479.

2. Amiel J, Lyonnet S. Hirschprung disease, associated syndromes, and genetics: a review. J Med Genet. 2001; 38 : 729-39.

3. Kessman J. Hirschsprung disease: diagnosis and management. Am Fam Physician. 2006; 74: 1319-22.

4. Puri P. Hirschsprung's disease: clinical generalities. In: Holschneider A, Puri P, editors. Hirschsprung's disease and allied disorders. Amsterdam: Harwood Academic Publishers; 2000. p. 120-35.

5. Hansford JR, Mullingan LM. Multiple endocrine neoplasia type 2 and RET: from neoplasia to neurogenesis. J Med Genet. 2000; 37: 817-27.

6. Simon S, Pavel M, Hensen J, Berg J, Hümer H-P, Carbon R. Multiple endocrine neoplasia 2A syndrome: Surgical management. J Pediatr Surg. 2002; 37: 897-900.

7. Gariepy CE. Genetic basis of Hirschsprung disease: implication in clinical practice. Mol Genet Metabol. 2003; 80: 6673.

8. Holscheneider A, Puri P. Hirschsprung's disease and allied disorders. Amsterdam: Harwood Academic Publishers; 2000 . funcional. Entretanto, o estudo minucioso caso a caso permitiu afirmar que, pelo menos, 18 pacientes apresentaram soiling, enterocolite recorrente, obstrução intestinal, diarréia, constipação severa, distensão abdominal, desnutrição e/ou distúrbios psicológicos, estes últimos podendo ser significativos e frequentes, como relatado por Athanasakos et $a l ., 20$ anos após a cirurgia definitiva e resultantes das experiências vividas em função da doença.

A associação da DH com síndromes genéticas deve ser considerada não só pela comorbidade, mas também porque muitas síndromes associadas não expressam o fenótipo completamente, podendo passar despercebidas, trazendo implicações para o prognóstico. ${ }^{2-7}$ Nos 22 pacientes da série rastreados para a presença de mutações genéticas, não foram identificadas mutações de ponto associadas a MEN2A e DH, sendo esse um resultado esperado, considerando-se principalmente a raridade da doença (MEN2A) e o número restrito de pacientes investigados. Ressalta-se, todavia, que a literatura especializada recomenda a tireoidectomia profilática na infância, acompanhamento por endocrinologista, e rastreamento de parentes de primeiro grau para que essas mutações sejam detectadas. 21,22

9. Schärli AF. Pathophysiology of classical Hirschsprung's disease. In: Holschneider A, Puri P, editors. Hirschsprung disease and allied disorders. Amsterdam: Harwood Academic Publishers; 2000. p. 109-125.

10. Nussbaum LR, McInnes RR, Willard HF. Thompson \& Thompson. Genetics in medicine. St. Louis: W. B. Saunders Company; 2001.

11. Brown RA, Cywes S. Disorders and congenital malformations associated with Hirschsprung's disease. In: Holschneider A, Puri P, editors. Hirschsprung's disease and allied disorders. Amsterdam: Harwood Academic Publishers; 2000. p. 137-45.

12. Langer JC, Durrant AC, de la Torre L, Teitelbaum DH, Minkes RK, Caty MG, Wildhaber BE, Ortega SJ, Albanese CT. One-stage transanal Soave pullthrough for Hirschsprung disease: a multicenter experience with 141 children. Ann Surg. 2003; 238: 569-83.

13. Singh SJ, Croaker GDH, Manglick P, Wong CL, Athanasakos H, Elliot E, Cass D. Hirschsprung's disease: The Australian Paediatric Surveillance Unit's Experience. Pediatr Surg Int. 2003; 19: 247-50.

14. Singh R, Cameron BH, Walton JM, Farrokhyar F, Borenstein SH, Fitzgerald PG. Postoperative Hirschsprung's enterocolitis after minimally invasive Swenson's procedure. J Pediatr Surg. 2007; 42: 885-9. 
15. Fonkalsrud EW. 2000. Complications of Hirschsprung's disease and allied disorders. In: Holschneider A, Puri P, editors. Hirschsprung's disease and allied disorders. Amsterdam: Harwood Academic Publishers; 2000.p. 42531 .

16. Tsuji H, Spitz L, Kiely EM, Drake DP, Pierrô A. management and long-term follow-up of infants with total colonic aganglionosis. J Pediatr Surg. 1999; 34: 158-61.

17. Bai Y, Chen H, Hao J, Huang Y, Shenyang WW. Long-term and quality of life after the Swenson procedure for Hirschsprung's Disease. J Pediatr Surg. 2002; 37: 639-42.

18. Engum SA, Grosfeld JL. Long-term results of treatment of Hirschsprung's disease. Semin Pediatr Surg .2004; 13: 27385

19. Menezes M, Corbally M, Puri P. Long-term results of bowel function after treatment for Hirschsprung's disease. Pediatr Surg Int. 2006; 22: 987-90.

Recebido em 21 de janeiro de 2009

Versão final apresentada em 15 de junho de 2009

Aprovado em 17 de junho de 2009
20. Athanasakos E, Starling J, Ross F, Nunn K, Cass D. An example of psychological adjustment in chronic illness: Hirschsprung's disease. Pediatr Surg Int. 2006; 22: 319-25.

21. Brandi ML, Gagel RF, Angeli A, Bilezikian JP, BeckPeccoz P, Bordi C, Conte-Devolx B, Falchetti A, Gheri RG, Libroia A, Lips CJ, Lombardi G, Mannelli M, Pacini F, Ponder BA, Raue F, Skogseid B, Tamburrano G, Thakker RV, Thompson NW, Tomassetti P, Tonelli F, Wells SA Jr, Marx SJ. Guidelines for diagnosis and therapy of MEN type 1 and type 2. J Clin Endocr Metab. 2001; 86: 5658-71

22. Ceccherini I, Attié T, Martuccielo G, Nihoul-Fékété C, Lyonnet S, Romeo G. 2000. The molecular genetics of Hirschsprung's disease. In: Holschneider A, Puri P, editors. Hirschsprung's disease and allied disorders. Amsterdam: Harwood Academic Publishers; 2000. p. 69-80. 